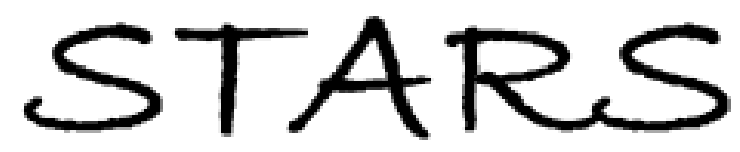

University of Central Florida

STARS

$1-1-2007$

\title{
Role of Brownian motion on the thermal conductivity enhancement of nanofluids
}

\author{
Amit Gupta \\ University of Central Florida \\ Ranganathan Kumar \\ University of Central Florida
}

Find similar works at: https://stars.library.ucf.edu/facultybib2000 University of Central Florida Libraries http://library.ucf.edu

This Article is brought to you for free and open access by the Faculty Bibliography at STARS. It has been accepted for inclusion in Faculty Bibliography 2000 s by an authorized administrator of STARS. For more information, please contactSTARS@ucf.edu.

\section{Recommended Citation}

Gupta, Amit and Kumar, Ranganathan, "Role of Brownian motion on the thermal conductivity enhancement of nanofluids" (2007). Faculty Bibliography 2000s. 7192.

https://stars.library.ucf.edu/facultybib2000/7192

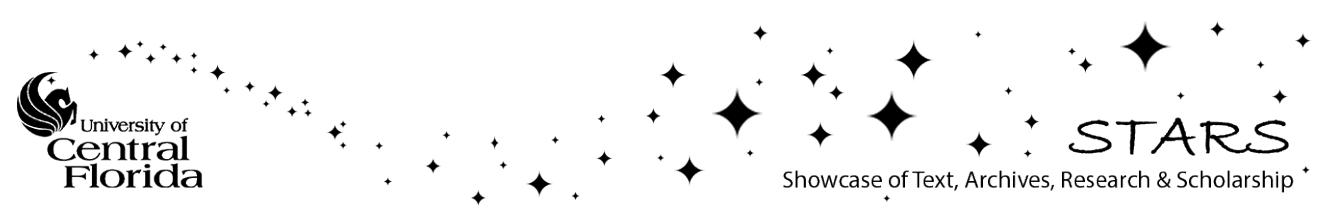




\section{Role of Brownian motion on the thermal conductivity enhancement of nanofluids}

Cite as: Appl. Phys. Lett. 91, 223102 (2007); https://doi.org/10.1063/1.2816903

Submitted: 13 October 2007 . Accepted: 01 November 2007 . Published Online: 26 November 2007

Amit Gupta, and Ranganathan Kumar

\section{ARTICLES YOU MAY BE INTERESTED IN}

Role of Brownian motion in the enhanced thermal conductivity of nanofluids

Applied Physics Letters 84, 4316 (2004); https://doi.org/10.1063/1.1756684

Role of Brownian motion hydrodynamics on nanofluid thermal conductivity

Applied Physics Letters 88, 093116 (2006); https://doi.org/10.1063/1.2179118

Anomalously increased effective thermal conductivities of ethylene glycol-based nanofluids containing copper nanoparticles

Applied Physics Letters 78, 718 (2001); https://doi.org/10.1063/1.1341218
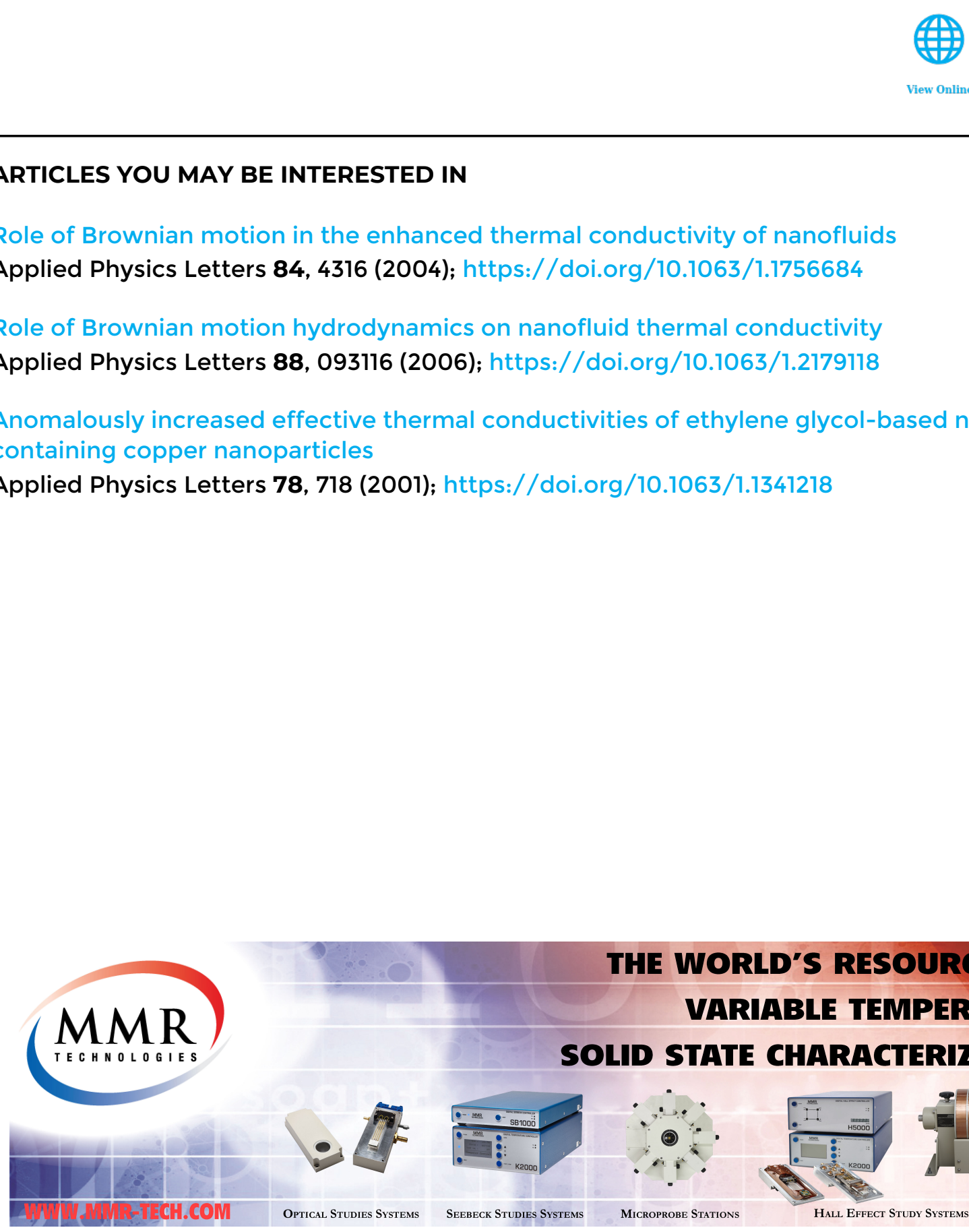


\title{
Role of Brownian motion on the thermal conductivity enhancement of nanofluids
}

\author{
Amit Gupta and Ranganathan Kumar ${ }^{\mathrm{a})}$ \\ Department of Mechanical, Materials and Aerospace Engineering, University of Central Florida, \\ Orlando, Florida 32816, USA
}

(Received 13 October 2007; accepted 1 November 2007; published online 26 November 2007)

\begin{abstract}
This study involves Brownian dynamics simulations of a real nanofluid system in which the interparticle potential is determined based on Debye length and surface interaction of the fluid and the solid. This paper shows that Brownian motion can increase the thermal conductivity of the nanofluid by $6 \%$ primarily due to "random walk" motion and not only through diffusion. This increase is limited by the maximum concentration for each particle size and is below that predicted by the effective medium theory. Beyond the maximum limit, particle aggregates begin to form. Brownian motion contribution stays as a constant beyond a certain particle diameter. (C) 2007 American Institute of Physics. [DOI: 10.1063/1.2816903]
\end{abstract}

A mixture of nanoparticles and base liquid called nanofluid has proved to be a highly effective method of enhancing heat transfer. Nanofluids possess high thermal conductivity and good suspension characteristics since the suspended crystalline solids and metals have thermal conductivities that are three orders of magnitude larger than that of the base liquids.

Thermal conductivity measurements of silica, copper, copper oxide, and aluminum oxide nanoparticles suspended in water and ethylene glycol have generated a lot of interest. Energy transport in such a nanofluid is affected not only by the size of the nanoparticle ${ }^{1}$ and the volume fraction of the dispersed medium but also on the $p \mathrm{H}$ of the solution. ${ }^{2}$ High effective conductivity has been measured in copper-ethylene glycol suspensions ${ }^{3}$ along with the modest enhancements for silver particles in water and toluene. ${ }^{4}$ Such high increases have been reported in the literature, however, there is no consensus on this issue. The discrepancies could very well be due to the differences in the nanofluid preparation, additives, size, and concentration of the particles.

It has been fairly well established that the high enhancements of thermal conductivity cannot be predicted by the Maxwell theory or its extension (Hamilton-Crosser model), which is applicable for colloidal particles of any shape such as sphere and cylinder. Several theories and hypotheses have been proposed that merit consideration. Although initially it was thought that the solidlike nanolayer around the particle may reduce the interfacial thermal resistance, molecular dynamics simulations did not show significant increase in nanofluid conductivity. ${ }^{5}$

Two other major mechanisms have been proposed to explain the anomalous increase in thermal conductivity: (1) Brownian motion ${ }^{6-12}$ and (2) particle aggregation. ${ }^{13}$ Langevin's equations were used in a true Brownian dynamics simulation. ${ }^{6}$ The parameters chosen in their interparticle potential were too small that yielded high nanofluid thermal conductivity. Other papers suggest that Brownian motion is only one of the mechanisms, and other mechanisms such as thermophoresis, ${ }^{8}$ microconvection, and temperature dependence $^{12,14}$ could be just as important. However, an op-

\footnotetext{
${ }^{a)}$ Electronic mail: Rnkumar@mail.ucf.edu.
}

posite view was taken ${ }^{9-11}$ that Brownian motion of the particles is too slow to transport a significant amount of heat through a nanofluid. The ratio of the Brownian motion contribution to thermal conductivity to the thermal conductivity of the base fluid was given by the ratio of the nanoparticle diffusivity to the fluid thermal diffusivity. ${ }^{10}$ Their analysis included only the diffusion and not the random motion of the particle, and concluded that the contribution from Brownian motion can utmost be $1 \%$. Their nonequilibrium molecular dynamics calculation showed that the maximum thermal conductivity enhancement was only $2.5 \%$ for $3.3 \%$ concentration, much less than predicted by effective medium theory. In another study, Monte-Carlo simulations enhanced conductivity by $25 \%$ for $4 \%$ concentration using fractal aggregates. $^{13}$

These findings suggest that Langevin's equations can be further exploited to understand the role of Brownian motion of colloidal particles at their maximum concentration prior to aggregation. The objective of this work is to quantify the effect of Brownian motion for real particles at different size and concentration in nanofluid suspensions by solving the Langevin form of the particle trajectory equation for silica nanoparticles suspended in water.

Ermak and McCammon ${ }^{15}$ developed a straightforward method of conducting a typical Brownian dynamics simulation. In their approach, the Langevin equations of motion are integrated over a time interval under the assumption that the systematic forces remain approximately constant. Ermak's algorithm is an attempt to treat both the systematic, dynamic, and stochastic elements of the Langevin equation. For long times, a steady state canonical distribution for the positions leads to $\mathbf{r}(t+\delta t)=\mathbf{r}(t)+\left(D / k_{B} T\right) \mathbf{f}(t) \delta t+\delta \mathbf{r}^{G}$, where $\mathbf{r}(t)$ is the position vector for the particle, $\delta t$ is the time step, $k_{B}$ is the Boltzmann constant, and $T$ is the temperature. $\delta \mathbf{r}^{G}$ is chosen from a Gaussian distribution with zero mean and variance such that $\left\langle\left|\delta \mathbf{r}^{G}\right|^{2}\right\rangle=2 D \delta$. In devising the algorithm for simulating the diffusive behavior of a system of $N$ interacting particles, the effects of hydrodynamic interactions mediated by the fluid are incorporated through an interparticle friction tensor $D .{ }^{15}$ The configuration space trajectories are calculated; the trajectories are composed of successive displacements each taken over $\delta t$. From the time evolution of the 


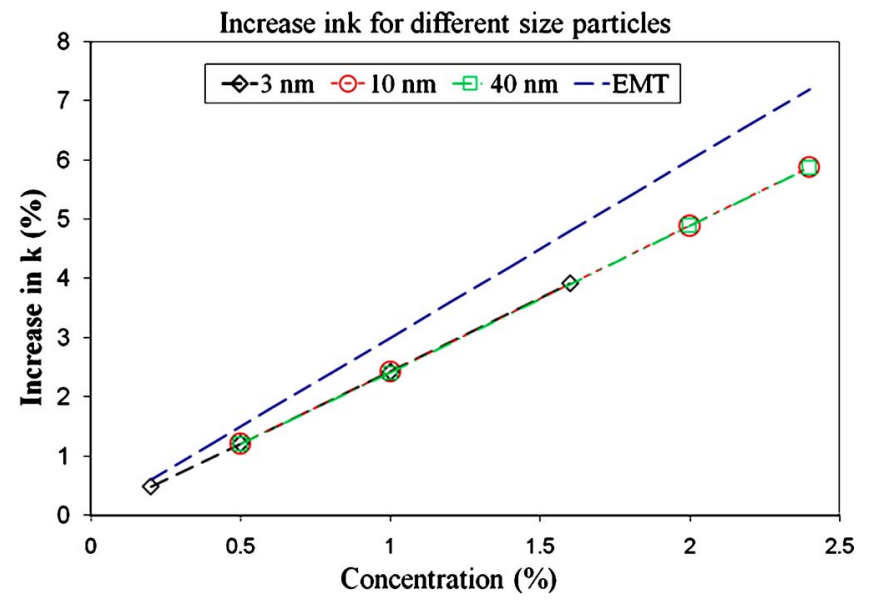

FIG. 1. (Color online) Increase in thermal conductivity for silica nanoparticles in water. The dotted line shows the maximum limit for the effective medium theory.

coordinates of the nanoparticles, the velocity of all the nanoparticles is calculated. This leads to the calculation of the "excess energy" $E_{i}$ of the particles. Calculating the heat flux operator $\mathbf{Q}(t)=(\partial / \partial t) \sum_{i} \mathbf{r}_{\mathbf{i}} E_{i}$, the thermal conductivity of the nanofluid is calculated using the Green-Kubo relation, $k_{n f}=\left[\sum_{j=0}^{n}\langle\mathbf{Q}(0) \mathbf{Q}(j \Delta t)\rangle \delta t\right] / k_{B} T^{2} V$, where $V$ is the volume of the domain and $n$ is the number of time steps used in the simulation.

A commonly used form of the interparticle potential is proposed by Elimelech ${ }^{16}$ is $\phi_{i j}=A \exp \left[-B\left(r_{i j}-d\right) / d\right]$, where $d$ is the diameter of the particles and $r_{i j}$ is the separation between the particle pair. A small value of $B$ would yield an unphysical constant interparticle force between a particle pair. ${ }^{6}$ The correct value of $B$ can be determined by considering the interparticle potential in a colloidal suspension, ${ }^{16}$ $\phi=\pi \varepsilon_{\text {per }} d \varphi^{2} \exp (-\kappa h)$, where $\varepsilon_{\text {per }}$ is the permittivity of the fluid, $\kappa$ is the Debye length, $\varphi$ is the zeta potential, and $h$ is the surface to surface separation, which when compared with the $\phi_{i j}$ expression yields $B=d \kappa$. The inverse Debye length, which is a measure of the double layer thickness, is of the order of a few atomic distances, i.e., $\sim 1 \mathrm{~nm} .{ }^{9,17}$ The sensitivity of minor changes in the double layer thickness to thermal conductivity was not found to be significant. The value of the parameter $A$ can also be found using the fluid property tables at any given temperature. For water, the potential expression for $10 \mathrm{~nm}$ sized silica particles would read as $\phi=2.22 \times 10^{-19} \exp (-\kappa h)$.

Several Brownian dynamics simulations were run for different particle sizes and concentrations with an initial configuration defined by a face-centered cubic arrangement and a time step of $10^{-9} \mathrm{~s}$, which is larger than the momentum relaxation time ${ }^{15}$ of $\sim 10^{-12}$ s. In Fig. 1, the nanofluid at $300 \mathrm{~K}$ shows an increase in the net thermal conductivity compared to the pure fluid which increases with particle concentration for different sizes. The conductivity due to Brownian motion is not negligible but not as high as the measured thermal conductivity, and is less than that predicted by the effective medium theory (EMT), with the deviation being high at high concentrations. EMT was calculated in the limit when there is no interfacial resistance to heat flow.

Simulations were also done for a range of particle sizes, from 1 to $50 \mathrm{~nm}$ in diameter, and at various volume concentrations and at different temperatures (Fig. 2). The maximum

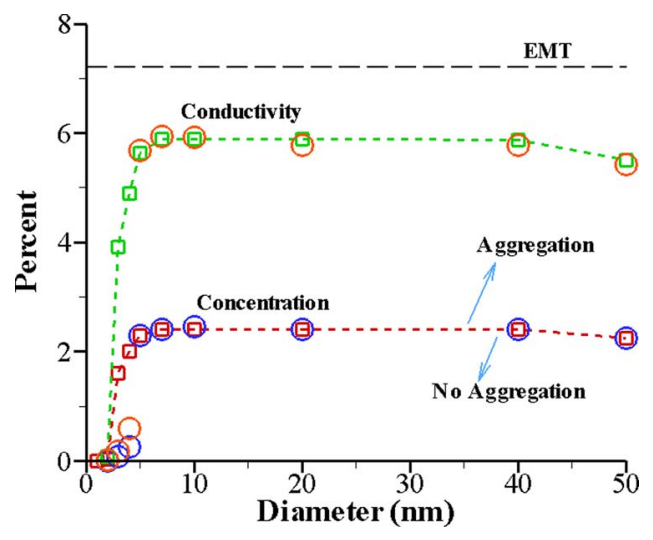

FIG. 2. (Color online) Maximum possible concentration and maximum thermal conductivity enhancement due only to Brownian motion as a function of the diameter of the nanoparticles at 300 (squares) and $350 \mathrm{~K}$ (circles). Particle aggregates begin to appear above the concentration line.

increase in thermal conductivity was found to be not more than $\sim 6 \%$, which occurred at high concentrations of the nanoparticles. EMT, which is constant at all diameters, provides the upper limit for comparison. Brownian velocity increased by a factor of 1.9 when the temperature was increased from 300 to $350 \mathrm{~K}$. But these velocities do not affect the maximum concentration up to which Brownian dynamics is valid or the nanofluid conductivity due only to Brownian motion of the nanoparticles. Very small particles $(1-5 \mathrm{~nm})$ show agglomeration at very low concentrations, which can be attributed to their high Brownian velocity and relatively higher particle diffusivity. As the particle size increases, the concentration curve flattens, above which particles begin to agglomerate (Fig. 3).

For very small nanoparticles, the random "walk" component of the particle displacement is considerable compared to the displacement associated with the particle diffusion because of the interparticle repulsive forces. This is because the random motion $\left|\delta \mathbf{r}_{G}\right|$ is proportional to the diffusion tensor $D^{1 / 2}$. In addition, $D \propto T \eta^{-1} d^{-1}$, where $\eta$ is the dynamic viscosity of the fluid. That makes the random walk to be proportional to $T^{1 / 2} \eta^{-1 / 2} d^{-1 / 2}$, which decays very rapidly as the particle size increases or the temperature decreases. So for a fixed temperature, between a 1 and $10 \mathrm{~nm}$ nanoparticles, this contribution is much higher for the former as compared to the latter. A similar argument holds for a particle of fixed size at 300 and $350 \mathrm{~K}$. On the other hand, the particle displace-

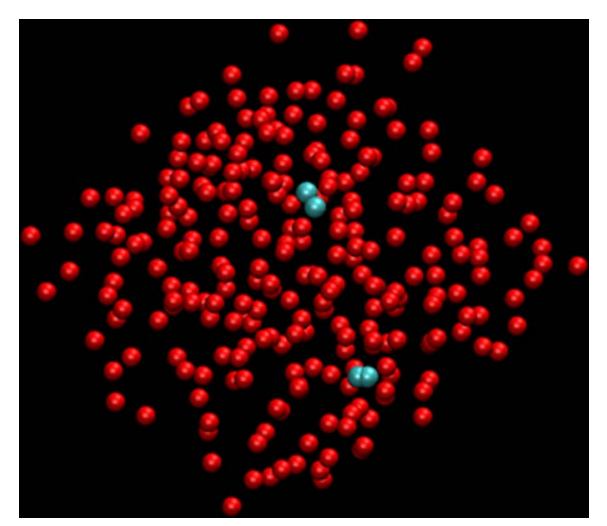

FIG. 3. (Color online) A snapshot of particle positions at slightly above the concentration limit where the behavior analogous to particle aggregation (in blue) is shown. 
ment due to systematic forces is $|\Delta \mathbf{r}| \propto D \mathbf{f}(t) / T$, where $\mathbf{f}(\mathrm{t})$ is the time-dependent force. The force is also proportional to the diameter of the nanoparticles because of the form of the interparticle potential used. So the displacement in the Langevin equation corresponding to the hydrodynamically mediated forces becomes proportional to $d \eta^{-1}$. Thus for small particles, this component is much smaller compared to that for large particles. Since the fluid viscosity decreases with an increase in temperature, the diffusion due to systematic forces for a small particle, is higher at higher temperatures. Thus, small nanoparticles $(1-5 \mathrm{~nm})$ have a high "random" diffusion exerted by other "like" nanoparticles in the vicinity. This lack of repulsion enables the formation of aggregates for such small particles at very low volume fractions, as seen from the interparticle distance becoming smaller than the diameter. Since the fluid viscosity decreases with an increase in temperature, the diffusion due to systematic forces for a small particle is higher at higher temperatures. On the other hand, large particles $(d \geqslant 10 \mathrm{~nm})$ have sufficiently high repulsion and low random diffusion. Beyond a critical volume fraction, agglomeration occurs not because of the random diffusion but due to smaller repulsion force between the nanoparticles (Fig. 3). Since agglomeration occurs at lower concentrations, Brownian motion alone cannot predict the increase in thermal conductivity. The appearance of aggregates in Fig. 3 at concentrations slightly over the limit begins to corroborate the Monte-Carlo results. ${ }^{13}$ The influence of morphology of the aggregates on thermal conductivity holds promise and needs to be explored further.

${ }^{1}$ D. Milanova and R. Kumar, Appl. Phys. Lett. 87, 233107 (2005).

${ }^{2}$ D. Milanova and R. Kumar, "Heat Transfer Behavior of Silica Nanoparticles in Pool Boiling Experiment," J. Heat Transfer (to be published).

${ }^{3}$ J. A. Eastman, S. U. S. Choi, S. Li, W. Yu, and L. J. Thompson, Appl. Phys. Lett. 78, 718 (2001).

${ }^{4}$ H. E. Patel, S. K. Das, T. Sundarajan, A. S. Nair, B. George, and T. Pradeep, Appl. Phys. Lett. 83, 2931 (2003).

${ }^{5}$ L. Xue, P. Keblinski, S. R. Phillpot, S. U. S. Choi, and J. A. Eastman, Int. J. Heat Mass Transfer 47, 4277 (2004).

${ }^{6}$ P. Bhattacharya, S. K. Saha, A. Yadav, P. E. Phelan, and R. S. Prasher, J. Appl. Phys. 95, 6492 (2004).

${ }^{7}$ S. P. Jang and S. U. S. Choi, Appl. Phys. Lett. 84, 4316 (2004).

${ }^{8}$ J. Buongiorno, J. Heat Transfer 128, 240 (2006).

${ }^{9}$ P. Keblinski, S. R. Phillpot, S. U. S. Choi, and J. A. Eastman, Int. J. Heat Mass Transfer 45, 855 (2002).

${ }^{10}$ W. Evans, J. Fish, and P. Keblinski, Appl. Phys. Lett. 88, 093116 (2006).

${ }^{11}$ M. Vladkov and J.-L. Barrat, Nano Lett. 6, 1224 (2006).

${ }^{12}$ D. H. Kumar, H. E. Patel, V. R. R. Kumar, T. Sundararajan, T. Pradeep, and S. K. Das, Phys. Rev. Lett. 93, 144301 (2004).

${ }^{13}$ R. S. Prasher, W. Evans, P. Meakin, J. Fish, P. Phelan, and P. Keblinski, Appl. Phys. Lett. 89, 143119 (2006).

${ }^{14}$ S. K. Das, N. Putra, P. Thiesen, and W. Roetzel, J. Heat Transfer 125, 567 (2003).

${ }^{15}$ D. L. Ermak and J. A. McCammon, J. Chem. Phys. 69, 1352 (1978).

${ }^{16} \mathrm{M}$. Elimelech, Particle Deposition and Aggregation: Measurement, Modeling and Simulation, 1st ed. (Butterworth-Heinmann, Oxford, 1995), pp. $38-42$.

${ }^{17}$ K. C. Leong, C. Yang, and S. M. S. Murshed, J. Nanopart. Res. 8, 245 (2006). 